\title{
Hacinamiento carcelario y estado de cosas inconstitucional en los centros penitenciarios. STC $\mathbf{N}^{\circ}$ 05436-2014-PHC/TC
}

\author{
Prison overcrowding and unconstitutional state of affairs in prisons. STC $\mathrm{N}^{\circ} 05436-2014-\mathrm{PHC} / \mathrm{TC}$ \\ Superlotação das prisões e estado de coisas inconstitucional nas prisões. STC Nº 05436-2014-PHC/TC
}

Artículo recibido en abril 2021

Arbitraje en mayo 2021

Aceptación en junio 2021

Publicación en julio 2021
Yully Isabel Chamorro Meza

isabelchm.13@gmail.com

https://orcid.org/ 0000-0001-9471-1054

Universidad César Vallejo, Lima-Perú
RESUMEN

ABSTRACT

RESUMO
El presente artículo, surge por la trascendencia que alcanzó el problema del hacinamiento carcelario con la sentencia del Tribunal Constitucional $N^{\circ}$ 05436-2014-PHC/TC, publicado el 19 de junio del 2020, que declaró como estado de cosas inconstitucional el hacinamiento de los establecimientos penitenciarios del Perú, toda vez que, como es de conocimiento público es un problema álgido que vulnera gravemente derechos fundamentales de las personas recluidas en los centros penitenciarios. Visto así, nos planteamos como objetivo del artículo realizar una revisión descriptiva de la bibliografía existente sobre ambas unidades. Por ello, se plantea como objetivo la interpretación de los derechos fundamentales en el marco constitucional peruano sobre el hacinamiento carcelario. Bajo la metodología de estudio de caso y análisis de fuente documental fenomenológica. En conclusión, en Perú el Tribunal Constitucional, a través de la STC $N^{\circ}$ 054362014-PHC/TC declaró como estado de cosas inconstitucional el problema del hacinamiento carcelario y otorgó al Estado un plazo de hasta el 2025, para que reduzca el hacinamiento.

Palabras clave: Hacinamiento carcelario; Estado de cosas inconstitucional; Establecimiento penitenciario; Derechos fundamentales

The present article arises due to the transcendence that the problem of prison overcrowding reached with the sentence of the Constitutional Tribunal $N^{\circ}$ 05436-2014-PHC/TC, published on June 19, 2020, which declared as unconstitutional the overcrowding of the penitentiary establishments of Peru, since, as it is of public knowledge, it is a critical problem that seriously violates the fundamental rights of the persons held in the penitentiary centers. Thus, the objective of the article is to carry out a descriptive review of the existing literature on both units. Therefore, the objective is the interpretation of fundamental rights in the Peruvian constitutional framework on prison overcrowding. Under the methodology of case study and phenomenological documentary source analysis. In conclusion, in Peru the Constitutional Court, through STC No. 054362014-PHC/TC declared as unconstitutional the problem of prison overcrowding and granted the State a deadline until 2025, to reduce overcrowding.

Key words: Prison overcrowding; Unconstitutional state of affairs; Prison; Fundamental rights

Este artigo surge da transcendência que o problema da superlotação das prisões atingiu com a sentença do Tribunal Constitucional N 05436-2014-PHC/TC, publicada em 19 de junho de 2020, que declarou como inconstitucional a superlotação das prisões no Peru, já que, como é do conhecimento público, é um grave problema que viola gravemente os direitos fundamentais das pessoas detidas nas prisões. Em vista disso, o objetivo do artigo é realizar uma revisão descritiva da bibliografia existente em ambas as unidades. Portanto, o objetivo do artigo é a interpretação dos direitos fundamentais no marco constitucional peruano sobre a superlotação das prisões. Sob a metodologia de estudo de casos e análise de fontes documentais fenomenológicas. Em conclusão, no Peru, o Tribunal Constitucional, através do STC N ${ }^{\circ}$ 054362014-PHC/TC declarou como inconstitucional o problema da superlotação das prisões e concedeu ao Estado um prazo até 2025, para reduzir a superlotação.

Palavras-chave: Superpopulação carcerária; Estado de coisas inconstitucional; Estabelecimento penitenciário; Direitos fundamentais 
INTRODUCCIÓN

El hacinamiento en Perú según informe de la Defensoría del Pueblo (2020) es la principal causa que perturban los DDHH del privado de libertad. Las tazas de hacinamiento alcanzan el $140 \%$ en la actualidad, en este País. Por lo que este organismo manifiesta que se deben realizar acciones urgentes a fin atender el grave riesgo que se incrementa en las poblaciones carcelarias según muestra el informe $\mathrm{N}^{\circ}$ 003-2020-DP, este además recomienda incrementar las posibilidades de acceso de conversión de pena, para aquellos delitos menores según denominación jurídica. También es importante destacar que el derecho a la salud de todas las personas privadas de libertad y de quienes los custodian, está plasmada dentro de la Constitución Política del Perú y bajo la normativa internacional y convenios suscrito.

Desde las evidencias, el objetivo de este artículo pretende la interpretación de los derechos fundamentales en el marco constitucional peruano desde el hacinamiento cancelario confinado en la realidad problemática corroborado con una interpretación de datos normativos ínsitos en el ordenamiento jurídico peruano, con el propósito de equiparar puntos de vista consensuados en defensa del ser humano.

\section{METODOLOGÍA}

El estudio se gestó sobre el enfoque cualitativo bajo el diseño metodológico de estudio de caso y análisis de fuente documental fenomenológica siendo la misma abordada desde una revisión bibliográfica de la normativa motivada virtualmente (Ludena, 2021).

Asimismo, fue de inducción y abducción analítica en su método, en tanto se trató de verificar teorías y proposiciones desde las investigaciones de carácter cualitativo a partir del surgimiento de fenómenos y constructos.

\section{DESCRIPCIÓN DE CASO}

Basado en los señalado, es importante analizar históricamente un proceso que dio origen al pronunciamiento del Tribunal Constitucional en Perú -el cual data desde el 11 de setiembre del año 2014, en donde manifiestan que un interno de iniciales C.C.B., del Establecimiento Penitenciario de Tacna, acudió ante el Juez de Tacna e interpuso demanda de Habeas Corpus contra las autoridades de dicho Establecimiento Penitenciario, alegando que se encontraba recluido desde el 21 de marzo del 2012 y padecía de enfermedades crónicas como bronquitis y gripe, las cuales no habían sido atendidos en su oportunidad, pese haber solicitado reiteradamente; por tal razón, el demandante argumentó que se habían vulnerado sus derechos a "la integridad personal y a la razonabilidad y proporcionalidad respecto de la forma 
y condiciones en que cumplía la pena". Añadió también que, por más de dos años se encontraba pernoctando en el suelo, hecho que había agravado el estado de su salud, por el contrario, frente a su pedido lo que recibía a cambio era un informe desfavorable de parte de la asistenta social, quien hacía alusión a que el demandante no asistía a las sesiones de seguimiento.

Por su parte, el Director del Establecimiento Penitenciario de Tacna, en su contestación de demanda, además de señalar que el demandante no tenía informes favorables debido a que no asistía al centro de estudios y trabajo, así como al seguimiento psicológico, social y legal, refirió también que dicho centro penitenciario se encontraba en una situación de hacinamiento, lo que impedía que los internos tengas camas individuales. Asimismo, el médico y la asistenta social del centro penitenciario, contestaron la demanda, aduciendo que el estado de salud del demandante era estable, pero que tiene antecedentes de haber sufrido tuberculosis pulmonar y que, en el 2012 no asistió a su tratamiento social, pero en el 2013 si cumplió, siendo falso las afirmaciones de que fue tratado mal por parte de las autoridades de dicho establecimiento.

Frente a tal escenario, el Juez del Primer Juzgado de Investigación Preparatoria de Tacna, el 16 de setiembre del año 2014, emitió sentencia declarando infundada la demanda al concluir que el demandante había recibido atención oportuna, que no había sido amenazada por la asistenta social y que no requería atención especializada conforme se podía verificar en la historia clínica.

El demandante por su parte, apeló dicha sentencia, la cual fue confirmada por la Sala Penal Superior de Tacna al considerar que las autoridades del centro penitenciario que no habían incumplido con sus funciones de brindar atención médica al demandante y que tampoco había evidencias de los malos tratos que alegaba por parte de la asistenta social.

Como última instancia, el demandante acudió vía recurso de agravio constitucional ante el Tribunal Constitucional, alegando que el Juez y los vocales de la Sala Superior, no habían verificado las condiciones en las que vivía y el trato diferenciado que existía, pues había internos que se encontraban en mejores condiciones que él. En tal sentido, el máximo intérprete de la constitución, después de haber analizado el tema del hacinamiento carcelario desde un ámbito general y considerando pronunciamientos anteriores, se pronunció: i) declarando fundada en parte la demanda; ii) declarando que existe un estado de cosas inconstitucional sobre el "permanente y crítico hacinamiento de los establecimientos penitenciarios y las severas deficiencias en la capacidad de albergue, calidad de su infraestructura e instalaciones sanitarias, de salud, de seguridad, entre otros servicios básicos, a nivel nacional."; iii) señalando que la solución al problema es el trabajo conjunto y coordinado entre los tres poderes del Estado, otras instituciones y la sociedad en general; iv) exhortando al Ministerio de Justicia y Derechos 
Humanos para que elabore un nuevo plan de política penitenciaria 2021-2025 a modo de política de Estado; v) señalando que si en el plazo de 5 años no se adoptaron medidas suficientes para abandonar dicho estado de cosas inconstitucional, los establecimientos penitenciarios podrían cerrarse temporalmente, impidiendo el ingreso de más internos y trasladando a los internos de las cárceles con hacinamientos a los que no tienen dicha condición, entre otras medidas que garanticen condiciones indispensables de permanencia en la cárcel, y de no mejorar tal situación empezaría por cerrarse los seis establecimientos más hacinados actualmente (Chanchamayo, Jaen, Callao, Camaná, Abancay y Miguel Castro Castro) o los que presenten tal situación en el 2025; vi) exhortando al Poder Judicial a que imponga prisión preventiva para aquellos delitos graves, que signifiquen un peligro social, mientras que para los otros delitos se aplique medidas alternativas; e, vii) indicando que la Comisión de Seguimiento y Cumplimiento de sentencias del Tribunal Constitucional realice audiencias públicas de supervisión cada seis meses.

\section{DISCUSIÓN DEL CASO}

Frente a los antecedentes fenomenológicos presentados en el estudio de caso de la referencia, cabe precisar dogmáticamente desde un diseño de teoría fundamentada que, en principio, el hacinamiento carcelario, es un problema de índole mundial y por tanto aqueja también a Perú, ello se evidencia en el Informe Estadístico del Instituto Nacional Penitenciario (INPE), emitido en febrero del 2020, que detalló que el $72 \%$ de los establecimientos penitenciarios (49), se encontraban en condición de hacinamiento, considerando que el porcentaje de sobrepoblación por cárcel se encuentra entre el $200 \%$ y el 600\%. Cárdenas (2020) en una entrevista para la revista el ojo público, manifestó que la sobrepoblación de las cárceles del país, es una dificultad crítica, debido a que los 68 establecimientos penitenciarios del país tienen capacidad para residir a 40 mil reos, entre varones y mujeres, empero la población asciende a 96 mil personas.

El problema se suscita en diversos ámbitos: en el contexto mundial, según Torres y Ariza (2019) el problema penitenciario, en muchos países, ocupó un lugar central en los debates académicos, jurídicos y políticos. En dicho debate se encuentra un factor importante que es el hacinamiento, por su parte, Alvarado y Vélez (2019) en un estudio que realizó con el objeto de contar con estadística actualizada sobre la realidad penitenciaria de varios países de América Latina y el Caribe, indicó que existe hacinamiento en los penales, ello se evidencia en la falta de las condiciones básicas para la convivencia del interno. En el contexto nacional, según el Informe Estadístico del Instituto Nacional Penitenciario (INPE, 2020), los 68 establecimientos penitenciarios del país tenían una población de 96,870 internos. Esta cifra evidencia un grave 
problema de hacinamiento. De dicho informe, se puede resaltar que, el $72 \%$, es decir 49 , se encuentran en condición de hacinamiento, considerando que el porcentaje de sobrepoblación por cárcel se encuentra entre el $200 \%$ y el $600 \%$.

El hacinamiento carcelario, viene a ser una situación en la cual, el aforo según el diseño original o la infraestructura de un establecimiento penitenciario es superado por un número mayor de internos que ingresan al mismo, conllevando a que se vulnere la dignidad de las personas que ocupan dichos espacios de reclusión, por ello, el autor propone que la problemática planteada sea definida desde tres dimensiones, como "capacidad instalada" referida a la capacidad de albergue, como "densidad de población" referida al espacio efectivo que tiene cada interno y como "estándar integral de alojamiento" que significa la existencia de un centro penitenciario que garantice los derechos fundamentales no restringidos por la privación de la libertad (Torres y Ariza, 2019). Asimismo, Cueva y Ruesta (2020), indica que el término, además de circunscribirse a un tema de aforo, también corresponde a diseños de política criminal y política penitenciaria como aristas de gestión pública del Estado.

El Tribunal Constitucional (TC, 2020) en la Sentencia N 05436-2014-PHC/TC, declaró como estado de cosas contra la constitución el hacinamiento de los establecimientos penitenciarios del Perú, otorgando al Estado un plazo de 05 años (que vence 2025) para que solucione dicha problemática, caso contrario se cerrarían los establecimientos penitenciarios que estén en dichas circunstancias, es decir, con la aludida sentencia se pretende exigir al Estado acciones inmediatas u urgentes que permitan solucionar un problema álgido que se presenta en casi todos los establecimientos penitenciarios de nuestro país, lo cual es un factor para la vulneración de los derechos de los internos, entre quienes se encuentran grupos vulnerables como ancianos, madres, niños, quienes como persona no han perdido el derecho a ser tratados con dignidad, por tanto a gozar de los derechos a la salud, la educación, el trabajo, la integridad física y mental, con el fin de resocializarse y retornar a la sociedad de la cual fueron relegadas por la comisión o presunta comisión de un ilícito penal. Pues bien, la realidad penitenciaria, pasa por el análisis de diversos factores que influyen en el panorama negativo que año a año se pone en evidencia, siendo por citar algunos, la insalubridad, la corrupción, la excesiva deshumanización de los penales, el aumento de penas, exceso de sanción, lo que ha conllevado a la sobrepoblación o hacinamiento de los diversos establecimientos penitenciarios de nuestro país.

Frente a ello, haciendo una revisión en la literatura internacional se evidencia que el hacinamiento carcelario al haber adquirido un carácter estructural y masivo, fue materia de intervención en el caso de Colombia, por la Corte Constitucional de dicho país, que, por primera vez, en la sentencia T-153 de 1998, declaró como estado de cosas inconstitucional. Este panorama, se replicó también en un pronunciamiento de la Corte Federal Suprema de Brasil, así 
como en nuestro país, en la sentencia N ${ }^{\circ}$ 5436-2014-PHC/TC (26-05-20), en el cual, el Tribunal Constitucional, declaró al hacinamiento carcelario como un estado de cosas inconstitucional.

En tal sentido, como medida de solución, el estudio del hacinamiento carcelario y el estado de cosas inconstitucional, va permitir establecer recomendaciones necesarias para de ser el caso seguir avanzando con la elaboración y emisión de políticas públicas para la solución del problema planteado, y en consecuencia lograr que los establecimientos penitenciarios alberguen a internos según la capacidad diseñada y con respeto a los derechos humanos y/o fundamentales de los internos, permitiendo de ese modo, una verdadera resocialización.

Luego de que un interno del establecimiento penitenciario de Tacna, interpuso demanda de habeas corpus (expediente $\mathrm{N}^{\circ}$ 05436-2014-PHC/TC-TACNA), por encontrarse con un tratamiento que carecía de razonabilidad y proporcionalidad en cuanto a la forma y condiciones en que cumple la pena, la misma que en primera y segunda instancia le fue denegada, el TC (2020) vía recurso de agravio constitucional, declaró fundada en parte la demanda y declaró como estado de cosas inconstitucional el hacinamiento carcelario de todos los establecimientos penitenciarios del país, otorgando al Estado un plazo de 5 años, a efectos de que a través de las diversas instituciones públicas involucradas en el problema, den solución.

Como parte, del cumplimiento de la sentencia del Tribunal Constitucional, el 25 de setiembre del 2020, fue publicada en el Diario Oficial El Peruano, el Decreto Supremo № 011-2020-JUS que aprueba la Política Nacional Penitenciaria al 2030.

\section{La declaratoria}

La técnica empleada por el Tribunal Constitucional, declarando como estado de cosas inconstitucional a problemas que se tornan estructurales, que afectan gravemente derechos fundamentales de un grupo o numerosas personas, no es nuevo, puesto que, desde el año 2004 hasta el 2020, ha emitido 14 sentencias, lo que a continuación se citan en la Tabla 1.

Tabla 1. Sentencias emitidas desde 2004-2020.

\begin{tabular}{ll}
\hline \multicolumn{1}{c}{ Sentencias } & \multicolumn{1}{c}{ Declaratoria } \\
\hline TC (2004) en el expediente 02579-2003-HD/TC, & Acceso a información en poder del CNM. \\
Caso Julia Eleyza Arellano Serquén. & \\
TC (2005) en el expediente 03149-2004-AC/TC, & $\begin{array}{l}\text { Ejecución de resoluciones que declaran un derecho } \\
\text { concedido en la Ley del Profesorado. }\end{array}$ \\
Caso Gloria Marleni Yarlequé Torres. & $\begin{array}{l}\text { Aplicación del principio de reserva de Ley en materia } \\
\text { tributaria. }\end{array}$
\end{tabular}


TC (2010a) en el expediente 05561-2007-AA/TC, Caso Oficina de Normalización previsional.

TC (2010b) en el expediente 0017-2008-PI/TC, Caso de la creación de filiales universitarias.

TC (2010c) en el expediente 03426-2008-HC/TC, Caso Pedro Gonzalo Marroquín Soto.

TC (2013) en el expediente 01722-2011-PA/ TC, Caso Sindicato de trabajadores de la Municipalidad de Lima-SITRAMUN.

TC (2014) en el expediente 01126-2012-PA/TC, Caso Dogner Lizith Díaz Chiscul.

TC (2016) en el expediente 02744-2015-PA/TC, Caso Jesús de Mesquita Oliviera y otros.

TC (2017a) en el expediente 04539-2012-AA/ TC, Caso Sindicato de trabajadores tributarios y aduaneros.

TC (2017b) en el expediente 00853-2015-PA/TC, Caso Marleni Cieza Fernández y otra.

TC (2018a) en el expediente 00889-2017-PA/TC, Caso María Antonia Díaz Cáceres de Tinoco.

TC (2018b) en el expediente 00799-2014-PA/TC, Caso Mario Eulogio Flores Callo.

TC (2019a) en el expediente 00009-2015-Al/TC, caso contra el DL 1133.

TC (2019b) en el expediente 00617-2017-AA/TC, Caso Marco Antonio Bocanegra Ruiz.
Participación temeraria, obstructiva y contraria a la jurisprudencia y precedentes por parte de los abogados de la Oficina de Normalización Previsional.

Derecho de acceso a una educación universitaria de calidad.

Política de tratamiento y rehabilitación de la salud mental de personas que se encuentran sujetas a medidas de seguridad de internación por padecer de una enfermedad mental.

Irrenunciabilidad de los derechos laborales.

La declaración de paternidad o maternidad no debe dar lugar a sanción administrativa en una institución educativa policial o militar.

Falta de una norma legal o reglamentaria que regule un procedimiento unificado, claro y específico, donde se precisen las garantías formales y materiales de los migrantes sujetos a un procedimiento migratorio sancionador.

Aplicación de sanciones por parte de la Sunat.

Disponibilidad y accesibilidad a la educación de personas en extrema pobreza del ámbito rural.

Derecho a que el Estado se comunique oficialmente también en lenguas originarias.

Ejercicio de las competencias por parte de la Oficina de Normalización Previsional.

Prohibición de doble percepción de ingresos para los pensionistas del Estado.

Tratamiento legislativo desigual en razón del sexo respecto a los requisitos para obtener pensión de viudez. 


\section{Discusión de la literatura}

Por lo que, el hacinamiento carcelario trae consigo la vulneración de derechos fundamentales de las personas recluidas en los establecimientos penitenciarios, haciendo que un penal se convierta en un lugar que no brinda las garantías mínimas en relación con los derechos humanos, ejemplo de ello, se evidencia en Colombia, en un estudio reporta que 19 internos murieron y 221 quedaron heridos, producto de las condiciones en las que se encuentran internados, los que son ocasionados por la deficiente infraestructura, el hacinamiento carcelario, los tratos inhumanos y crueles (Fajardo Sánchez, 2018), esto mismo ocurre en Brasil, pues los establecimientos carcelarios son lugares, en los que no se respeta las garantías mínimas de la dignidad humana (Perez, 2020).

El hacinamiento carcelario es uno de los fenómenos más complejos y graves por cuanto significa la pérdida de derechos de los internos, en cuanto a salud, integridad personal, educación, que además ocasiona y mantiene la corrupción como mecanismo para sobrevivir, lo que limita y hace casi imposible el proceso de resocialización (Gómez, 2015), esta tesis, es apoyada también con el estudio realizado por (Salazar y Medina, 2019), quien señala que en Colombia existen políticas de tratamiento para la reeducación y reinserción del interno a la sociedad, pero se ve limitado por el problema del hacinamiento.

El hacinamiento tiene causas como el populismo punitivo, la ineficiente política criminal y penitenciaria, esto conforme lo señaló el Tribunal Constitucional (TC, 2020) en la reciente sentencia STC $\mathrm{N}^{\circ}$ 05436-2014-PHC/TC, por ello, una solución, tal como se adoptó en otros países, sería la declaratoria del estado de cosas inconstitucional. Tesis que tiene aceptación en la comunidad jurídica, tal como se puede observar en el estudio realizado por (Cueva y Ruesta, 2020), quien asevera que ayudaría a mejorar la situación carcelaria de los internos.

El hacinamiento carcelario se ha tornado grave, recurrente y estructural, requiriendo y exigiendo de un tratamiento en gran escala por parte de las entidades públicas competentes, por ello, en la jurisprudencia y doctrina comparada, se pudo encontrar en Colombia diversas sentencias emitidas desde el año 1998 hasta el 2017, declarando como estado de cosas inconstitucional al problema citado, lo que ha generado diversos estudios sobre su efectividad y resultados de dicha práctica judicial, como ejemplo, se tiene el estudio de (Mondragón et. al, 2020) en Colombia y de (Orbage de Britto y Costa, 2019) en Brasil, quienes indican que el análisis del hacinamiento carcelario a la luz de la tesis desarrollado en Colombia, les permitió determinar el problema como carácter estructural y masivo. 
El problema planteado tiene diversas causas, como el populismo punitivo, la ineficiente política criminal y penitenciaria (STC N 05436-2014-PHC/TC), lo que requiere ser tratado a través de políticas públicas que las diversas entidades deben diseñar, luego de ser declarado como un estado de cosas inconstitucional, pues de lo contrario, se corre el riesgo de llegar a resultados como el que presenta (Fajardo, 2018) y (Salazar y Medina, 2019) en Colombia, en el que, el problema carcelario viene siendo tratado desde una óptica estructural, desde el año 1998, en el cual se declaró por primera vez, un estado contrario a la constitución.

Respecto a la eficacia de la declaratoria por parte del Tribunal Constitucional, como un estado de cosas inconstitucional al problema del hacinamiento carcelario, se podrá advertir de forma gradual, puesto que otorgó a las diversas instituciones públicas competentes un plazo de hasta el 2025, para terminar con dicha problemática, por tal razón no se tiene estudios con resultados positivos o negativos de tal tratamiento, ello se evidencia en el estudio de (Cueva y Ruesta, 2020), quien empíricamente concluyó en que la sentencia avizora resultados positivos.

La declaratoria del estado de cosas inconstitucional a problemas recurrentes y masivos se ha convertido en una práctica que permite inferir un posible resultado positivo, aunque tome años en alcanzar la finalidad, es por ello que, en Colombia, el estudio realizado por (Giraldo, 2020) pretende que el problema de la desatención a las cárceles de mujeres considerando del género sea visto también desde las aristas de dicha técnica judicial.

\section{CONCLUSIÓN}

El hacinamiento carcelario vulnera los derechos fundamentales no restringidos de las personas privadas de libertad, como la salud, integridad física y mental. Por lo que son varias las causas o factores que contribuyen al hacinamiento carcelario, entre ellas, se determinó la deficiente política criminal y penitenciaria, lo que favorece el incremento de la población carcelaria y disminuye la posibilidad de una eficaz política de reinserción del interno a la sociedad.

En Perú el Tribunal Constitucional, a través de la STC Nº54362014-PHC/TC declaró como estado de cosas inconstitucional el problema del hacinamiento carcelario y otorgó al Estado un plazo de hasta el 2025, para que reduzca el hacinamiento.

Además, se concluye que la existencia de instrumentos internacionales relacionados con la protección de los derechos humanos, no ha sido óbice para que los derechos de las personas privadas de libertad se vulneren.

Frente a la creciente problemática en Perú con el hacinamiento y según mostró el análisis de la literatura se pudo que Colombia haciendo uso de la técnica del Estado de Cosas Inconstitucional 
requirió la intervención del Estado a través de sus diversas instituciones competentes, a efectos que diseñen políticas públicas efectivas para contrarrestar el problema.

El Estado de Cosas Inconstitucional, exige para su declaratoria, la existencia masiva y generalizada de la vulneración de diversos derechos fundamentales, que afectan a un gran número de personas; el incumplimiento prolongado por parte de las autoridades de sus obligaciones de garantizar su derecho, tal como ocurre en el tema de la política criminal y penitenciaria.

En países como Colombia y Brasil, la aplicación de la técnica del Estado de Cosas Inconstitucional al problema carcelario, ha sido relativamente positivo, pues se ha logrado mejorar las condiciones de los internos.

No basta con construir más cárceles o con mejores infraestructuras para reducir el hacinamiento carcelario, sino que debe ser vista desde el punto de vista de los derechos humanos.

\section{REFERENCIAS}

Alvarado, N., y Vélez, V. (2019). Encuestas dentro de la cárcel: Una mirada al otro lado de las rejas. Proyecto del Banco Interamericano de Desarrollo. http://dx.doi. org/10.18235/0001858

Cárdenas, C. (2020). Entrevista en la Revista el ojo público. https://ojopublico.com/1622/datode-presidente-del-inpe-sobre-presos-sin

Cueva, W., y Ruesta, I. (2020). El Hacinamiento Carcelario y el Estado de Cosas Inconstitucional. Revista Jurídica Científica. https://doi.org/10.26495/rcs.v13i1.1297

Defensoría del Pueblo (2020). Situación de las personas privadas de libertad a Propósito de la declaratoria de emergencia sanitaria. Serie Informes Especiales $N^{\circ}$ 03-2020-DP

Decreto Supremo $N^{\circ}$ 011-2020-JUS (2020, 23 de setiembre). Presidente de la República. Diario Oficial El Peruano. https://www.gob.pe/ institucion/minjus/normas-legales/1477026011-2020-jus

Fajardo, L. (2018). Derechos Humanos de Personas Privadas de la Libertad en Colombia. Revista Republicana. http://ojs.urepublicana.edu. co/index.php/revistarepublicana/article/ view/452/398
Giraldo, K. (2020). Cárceles para mujeres: la necesidad de implementar el enfoque de género en el proceso de superación del estado de cosas inconstitucional en materia penitenciaria y carcelaria en Colombia. Estudios De Derecho. https://revistas.udea. edu.co/index.php/red/article/view/342877

Instituto Nacional Penitenciario (2020). Informe Estadístico (02-20). https://www.inpe.gob.pe/ normatividad/documentos/4369-informeestadistico-febrero-2020/file.html

Mondragón, S., Guzmán, A. y Pérez, A. (eneroabril, 2020). Regulación del tratamiento penitenciario y carcelario en el marco de los derechos humanos. Revista Virtual Universidad Católica del Norte. https://doi. org/10.35575/rvucn.n59a10

Orbage de Britto, E.,yCosta,W.(2019). Dialógo entre Colombia E Brasil Sobre O "Estado De Coisas Inconstitucional". Revista de la Secretaría del Tribunal Permanente de Revisión, 7(13), 193212. http://dx.doi.org/10.16890/rstpr.a7.n13. p193

Perez, S. (2020). El Covid-19 y la Crisis Carcelaria en Tiempos De Pandemia: una perspectiva desde el panorama penitenciario en Brasil. Revista Da Seção Judiciária Do Rio de Janeiro. https:// 
doi.org/https://doi.org/10.30749/21778337. v24n49p173-197

Salazar, W., y Medina, R. (2019). Estándares nacionales e internacionales del tratamiento penitenciario y carcelario en Colombia. Un estudio del caso de la cárcel de Neiva (Huila). Revista Prolegómenos, 22(43). https://doi. org/10.18359/prole.3460

STC $N^{\circ}$ 02579-2003-HD/TC (2004, 06 de abril). Tribunal Constitucional (Caso Julia Eleyza Arellano Serquén). https://www. tc.gob.pe/jurisprudencia/estado-de-cosasinconstitucional/

STC N ${ }^{\circ}$ 03149-2004-AC/TC (2005, 20 de enero). Tribunal Constitucional (Caso Gloria Marleni Yarlequé Torres) https://www. tc.gob.pe/jurisprudencia/estado-de-cosasinconstitucional/

STC $N^{\circ}$ 6626-2006-PA/TC (2007, 19 de abril). Tribunal Constitucional (Caso Importadora y Exportadora A.S. S.C.R.L) https://www. tc.gob.pe/jurisprudencia/estado-de-cosasinconstitucional/

STC N 05561-2007-AA/TC (2010, 24 de marzo). Tribunal Constitucional (Caso Oficina de Normalización previsional) https://www. tc.gob.pe/jurisprudencia/estado-de-cosasinconstitucional/

STC N ${ }^{\circ}$ 0017-2008-PI/TC (2010, 15 de junio). Tribunal Constitucional (Caso de la creación de filiales universitarias) https://www. tc.gob.pe/jurisprudencia/estado-de-cosasinconstitucional/

STC N ${ }^{\circ}$ 03426-2008-HC/TC (2010, 26 de agosto). Tribunal Constitucional (Caso Pedro Gonzalo Marroquín Soto). https://www. tc.gob.pe/jurisprudencia/estado-de-cosasinconstitucional/

STC N $N^{\circ}$ 01722-2011-PA/TC (2013, 11 de julio). Tribunal Constitucional (Caso Sindicato de trabajadores de la Municipalidad de Lima-SITRAMUN). https://www.tc.gob. pe/jurisprudencia/estado-de-cosasinconstitucional/

STC N 01126-2012-PA/TC (2014, 06 de marzo) Tribunal Constitucional (Caso Dogner Lizith Díaz Chiscul). https://www.tc.gob. pe/jurisprudencia/estado-de-cosasinconstitucional/
STC N $N^{\circ}$ 02744-2015-PA/TC (2016, 08 de noviembre). Tribunal Constitucional (Caso Jesús de Mesquita Oliviera y otros). https:// www.tc.gob.pe/jurisprudencia/estado-decosas-inconstitucional/

STC Nº0853-2015-PA/TC (2017, 14 de marzo). Tribunal Constitucional (Caso Marleni Cieza Fernández y otra). https://www. tc.gob.pe/jurisprudencia/estado-de-cosasinconstitucional/

STC No 04539-2012-AA/TC (2017, 20 de junio). Tribunal Constitucional (Caso Sindicato de trabajadores tributarios y aduaneros). https:// www.tc.gob.pe/jurisprudencia/estado-decosas-inconstitucional/

STC N ${ }^{\circ}$ 00889-2017-PA/TC (2018, 17 de abril). Tribunal Constitucional (Caso María Antonia Díaz Cáceres de Tinoco). https://www. tc.gob.pe/jurisprudencia/estado-de-cosasinconstitucional/

STC $\mathrm{N}^{\circ}$ 00799-2014-PA/TC (2018, 05 de diciembre). Tribunal Constitucional (Caso Mario Eulogio Flores Callo). https://www. tc.gob.pe/jurisprudencia/estado-de-cosasinconstitucional/

STC N ${ }^{\circ}$ 00009-2015-Al/TC (2019, 19 de abril). Tribunal Constitucional (Caso contra el DL 1133). https://www.tc.gob.pe/jurisprudencia/ estado-de-cosas-inconstitucional/

STC $\mathrm{N}^{\circ}$ 00617-2017-AA/TC (2019, 18 de noviembre). Tribunal Constitucional (Caso Marco Antonio Bocanegra Ruiz).https://www. tc.gob.pe/jurisprudencia/estado-de-cosasinconstitucional/

STC No 05436-2014-PHC/TC (2020, 19 de junio). Tribunal Constitucional (Caso Hacinamiento de los penales y deficiencias de albergue, en la calidad de su infraestructura, instalaciones sanitarias, salud, seguridad y servicios básicos a nivel nacional). https://www. tc.gob.pe/jurisprudencia/estado-de-cosasinconstitucional/

Torres, A., y Ariza, L. (2019). Definiendo el hacinamiento. Estándares normativos y perspectivas judiciales sobre el espacio penitenciario. Estudios Socio-Jurídicos. https:// doi.org/10.12804/revistas.urosario.edu.co/ sociojuridicos/a.7632 